\title{
ONTOLOGY ENRICHMENT FOR MULTI-DOMAIN KNOWLEDGE AND EXPERTISE REPRESENTATION
}

\author{
Harry T Yani Achsan, Heru Suhartanto \& Wahyu Catur Wibowo
}
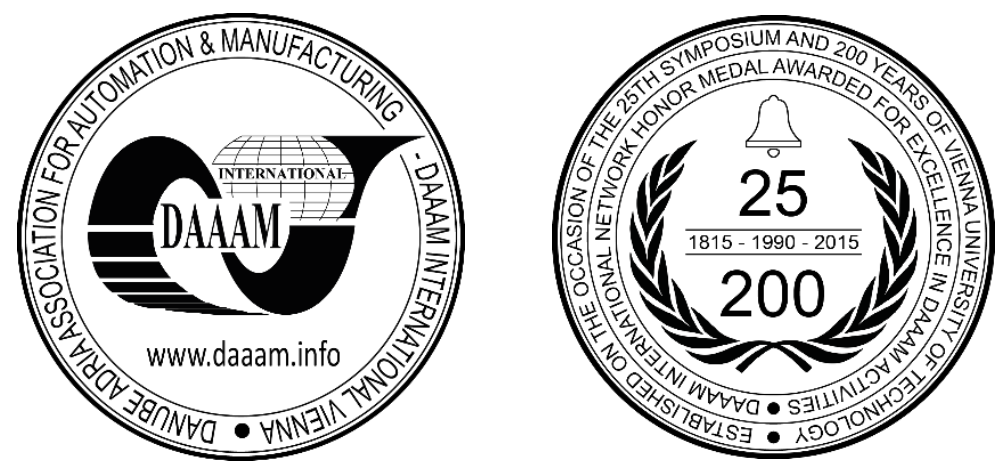

This Publication has to be referred as: Achsan, H[arry] T[. Yani]; Suhartanto, H[eru] S[eok] \& Wibowo, W[ahyu] C[atur] (2017). Ontology Enrichment for Multi-Domain Knowledge and Expertise Representation, Proceedings of the 28th DAAAM International Symposium, pp.1170-1177, B. Katalinic (Ed.), Published by DAAAM International, ISBN 978-3-902734-11-2, ISSN 1726-9679, Vienna, Austria

DOI: $10.2507 / 28$ th.daaam.proceedings. 162

\begin{abstract}
Expertise is specialized skills and knowledge. It can be shared or disseminated if stated explicitly using expertise representation. Since people have multi area/domain expertise, we need multi-domain expertise representation. Someone's expertise can be recognized by his/her publication. In this article we proposed a new expertise representation by utilizing bibliography (publication metadata) and annotating ontology (ontology enrichment). The expertise representation is multi-domain that can be integrated semantically as easy as single-domain ontology.
\end{abstract}

Keywords: expertise; knowledge representation; semantic heterogeneity; multi-domain ontology; bibliography; library catalogue

\section{Introduction}

Tacit knowledge cannot be transferred to other people unless it is stated explicitly. Since stone age many media have been developed to state knowledge explicitly e.g. slate, leather, paper, microfiche, magnetic tape, magnetic disk, optical disk, flash disk, solid state disk, etc. Some media can be used to transfer knowledge directly like slate, leather and paper, but some other media can transfer knowledge by using analogue or digital equipment only.

Recently, the trend of transferring knowledge uses digital environment [1]. Storing and transferring knowledge digitally has many advantages; one of them is that the knowledge stored digitally is not only can be presented to human but also can be processed by machine. It means that the machine has the ability to read and to interpret knowledge to be manipulated, synthesized, and presented.

In supporting and improving readability, the digitally stored knowledge should be understood by machine. Unfortunately, most of knowledge are represented in such a way that human can read easily and not intended for machine to understand it. Tim Berners-Lee, the founder of WWW, stated that the goal of WWW was not only for human-human communication, but also for machines to be able to participate and help. Thus, he proposed the Semantic Web two decades ago [2]. It is an extension of HTML that enables description of contents to be machine-readable and was developed based on Ontology [3]. Therefore, it can be inferred that ontology is a knowledge representation intended for both human and machine. 
Ontology is now used widely in many domains like in health $[4,5,6]$, engineering $[7,8]$, software $[9,10]$, commerce $[11,12,13]$, law $[14,15]$, etc. Those Ontologies use different Upper Ontology. Consequently, reasoning with many different domains of Ontology cannot be done automatically since it is labour intensive and costly.

Reasoning is the process of thinking about something in a logical way in order to form a conclusion or judgment [16]. One important goal of constructing Ontology is to synthesize new knowledge by using reasoning. Reasoning of Ontology in a multi-domain environment (a.k.a. semantic heterogeneity) has to be done manually because it needs Ontology merging $[17,18,19]$. Ontology merging is an intentional process of creating new ontology by mapping and matching concepts from two Ontologies or more.

Ontology merging from multi-domain needs multi-domain Ontology, a multi-domain knowledge representation. By using multi-domain Ontology, merging of multi-domain Ontology can be treated as single-domain/domain-specific Ontology merging that can be done automatically/semi-automatically.

This paper proposed novelty knowledge representation in multi-domain environment by utilizing Ontology enrichment. Ontology enrichment is annotating Ontology with other metadata. The metadata used here are library classification codes. These codes are used to classify books, journals, proceedings, and other library items.

Library items are authored by experts. An expert is a person who has extensive skill or knowledge in a particular field [20]. Since library items contain expertise (a written opinion by an expert [21]), the enriched Ontology can also be used to represent expertise.

\section{Related Research Work}

\subsection{Knowledge representation}

Several knowledge representations have been developed since the emergence of computer. Early knowledge representation that uses modern notation is Classical logic, one of Formal Logical Systems. Some members of classical logic which are still being used up until now are Propositional Logic [22, 23, 24], First-Order Logic [25, 26, 27, 28], and Second-Order Logics [29, 30, 31] among others. These logical systems are still being used and developed because their capability in knowledge reasoning. Unfortunately, these knowledge representations are easily read by mathematician but not for others.

Some graphical knowledge representations have been developed for people who are not familiar with mathematics notations. Graphical knowledge representations have gained attention by researchers after Margareth Masterman introduced Semantic Network in 1961 [32]. Semantic Network are implemented in many research areas like Brain and Cognition [33, 34, 35], Health Care [36, 37], Education [38, 39], Communication [40], Computer Science [39, 41, 42], etc. Other graphical knowledge representations are: Association Diagram introduced by Rakesh Agrawal [43, 44], Conceptual Graph introduced by John F. Sowa [45], Tree Diagram, and Bayesian Networks.

Graphical knowledge representations have some advantage over classical logics since they can be understood by "common people" and can be interpreted faster. However, it also has a big drawback since it was not designed for computation. Modern knowledge representation should be designed for human and for machine. Therefore, it enables machine to process the knowledge, increasing its versatility in many applications like: coal mining [46], information security [47], medical [48], user profiling [49], and product designing [50].

\subsection{Ontology}

Ontology is a term in computer science adopted from philosophy. In philosophical study, ontology is about existence. In computer science, an ontology is an explicit specification of a conceptualization [51]. The term ontology used in computer science began when Amitabha Ghose wrote articles about semantic of programming languages [52, 53] in 1970s. Ontology for knowledge representation and knowledge sharing introduced about two decades later by Thomas R. Gruber [51], Nicola Guarino [54], O'Leary [55], etc.

Ontology is categorized by Nicola Guarino into Upper/Top Level Ontology, Domain Ontology, Task Ontology, and Application Ontology. Top Level Ontology describes general concepts which are independent of specific domain. Domain Ontology and Task Ontology specialize concepts found in the Upper Ontology into to a generic domain or task respectively. Application Ontology describe concepts introduced by both Domain and Task Ontology.

Ontology as a knowledge representation is intended to share common understanding among people or software agents $[56,57]$. The most successful implementation of ontology as a knowledge representation is Semantic Web although the progress is very slow. At least 2.5 billion web pages have implemented Semantic Web in a sample of over 12 billion web pages, but it was only 21 percent [58]. Even though the progress is very slow, individuals and organizations insist to still implement Semantic Web, because it can decrease the page viewed per download by $50 \%$ and increase revenue by $20 \%$ [59].

Based on 627 surveys conducted by Jorge Cardoso in 2007 [59], he found that mostly people (68.2\%) used Protégé as their ontology editor. Ontology languages mostly used were OWL \& RDFS (75.9\% \& 64.9\% respectively), while most used reasoning engine was Jena (53.6\%). He also discovered that industries represented their knowledge with ontologies were mostly Education (31.0\%), Computer and Software (28.5\%), and Government (17\%). Most of them (60.0\%) did not employ any methodology or method to develop their ontologies. 
The next finding was about the purpose of developing ontologies. Majority of participants (69.9\%) used ontologies to share common understanding of the structure of information among people and software agents, and some $56.3 \%$ use ontologies to enable reuse of domain knowledge.

Two-third of surveyed subjects used ontology mapping to manipulate ontologies. Ontology mapping is a process of relating two terms from two ontologies that has the same meaning. Ontology mapping is crucial part in semantic integration, a process of interrelating information from diverse sources [60].

\subsection{Expertise}

Expertise is part of person profile, facts about someone. It can be developed by: a) web log analysis using Association Rule [61], fuzzy classification [62], and fuzzy clustering [63, 64], b) semantic enrichment on twitter messages and on emails, and c) tags analysis of social media. Since expertise is specialized skills and knowledge [64], it can be expressed as knowledge representation.

\section{Bibliography}

\subsection{Metadata}

Metadata is data to describe other data. Bibliography is metadata of library items. Bibliography in scholarly work is a list of books consisting metadata of books: the book title, year published, authors name, etc. Metadata of book can be found in libraries, specifically in the catalogue cards. Recently, most librarians do not use catalogue cards anymore since they have digitalized them. Searching library items is more convenient and more effective by using digitalized catalogue.

\subsection{Sources}

There are many sources of bibliography in the digital era. Online book stores provide bibliography of the items they sell. School and higher education libraries also serve online catalogue to search their items. Other sources of online catalogues are bibliographic databases like DOAJ (Directory of Open Access Journals), Scopus, and WOS (Web of Science). The amount of online catalogue or bibliography is very large. For example, a library can hold more than a million records. Scopus, a bibliographic database, hold more than 55 million records [61]. The largest bibliographic database is WorldCat that holds 2 billion items [62].

\subsection{Mining}

Collecting bibliography is very exhaustive and resource intensive if done manually because of its enormous size. It must be done automatically by using focused web-crawler that can collect data much faster than human can do manually [63].

\subsection{Classification}

Book classification has been employed in library for centuries. There are two major classification used by librarians all around the world which are Library of Congress Classification (LCC) and Dewey Classification Code (DCC). DDC is the most adopted classification code, but most of large libraries implement LCC because LCC uses alphanumeric and DDC uses numeric codes. Therefore, LCC has much more classes for the same length of codes. This is the reason why this paper focused on LCC.

Those book classifications are organized hierarchically such as tree diagram. There is no root node (level 0) because the root node is virtual. The first code is alphabetic, the second is alphanumeric, and the rest are numeric. There are 21 LCC nodes on level 1; some are mnemonics, like G (Geography), M (Music), P (Philology), and T (Technology). Level 2 has 213 nodes, where node M (Music) has 2 nodes on level 2: ML (Literature on music) and MT (Instruction and study), and node $\mathrm{T}$ has 16 children on level 2. Each level 1 node can be seen as representation of a domain, but a domain can be represented in more than one level 1 nodes. Nodes $\mathrm{C}$ to $\mathrm{F}$ are about history.

\subsection{Semantics}

A word in one domain can have different meaning in another domain. The word "conductor" have four meanings depends on the domain (the meanings were taken from WordNet, a lexical database), see Table 1. A word that has multiple meanings is called polysemy, and the science which study about meaning of words in a language is semantics. 


\begin{tabular}{|c|c|c|c|}
\hline Domain & Meaning & & LCC Codes \\
\hline \multirow{3}{*}{ Music } & \multirow{3}{*}{$\begin{array}{l}\text { The person who leads a } \\
\text { musical group }\end{array}$} & M1-5000 & Music \\
\hline & & MT1-960 & Instruction and study \\
\hline & & MT85 & Conducting. Score reading and playing \\
\hline \multirow{4}{*}{ Physics } & \multirow{4}{*}{$\begin{array}{l}\text { A substance that readily } \\
\text { conducts e.g. electricity and } \\
\text { heat }\end{array}$} & QC1-999 & Physics \\
\hline & & QC251-338.5 & Heat \\
\hline & & QC310.15-319 & Thermodynamics \\
\hline & & QC501-766 & Electricity and magnetism \\
\hline \multirow{3}{*}{ Transportation } & \multirow{3}{*}{$\begin{array}{l}\text { The person who collects } \\
\text { fares on a public conveyance }\end{array}$} & G1-922 & Geography (General) \\
\hline & & GT1-7070 & Manners and customs (General) \\
\hline & & GT5220-5286 & Customs relative to transportation and travel \\
\hline \multirow{2}{*}{ Electronics } & \multirow{2}{*}{$\begin{array}{l}\text { A device designed to } \\
\text { transmit electricity, heat, etc. }\end{array}$} & TK1-9971 & Electrical engineering. Electronics. Nuclear \\
\hline & & TK3001-3521 & Distribution or transmission of electric power \\
\hline
\end{tabular}

Table 1. The meanings of "conductor" in some domains and its corresponding LCC codes.

Table 1 shows the corresponding LCC classes of the word related to its domain. Since every book in library should be classified and the class of a book can be related to its domain, therefore the domain of the book can be known. Thus, a book of an academic journal or a conference proceeding should consist of papers for the same domain. For example, the proceeding of DAAAM (Danube Adria Association for Automation \& Manufacturing) contains papers in the domain of Automation \& Manufacturing only, and Nature Medicine journal consist articles in the domain of medicine only.

Semantically, the meaning of words can be known if the class of the book that contains the words is stated. For instance, a journal with LCC code ML468 must be Music Literature in the domain of music and more specifically about instruments and instrumental music. The domain can be found by searching code ML in M-outline.pdf file that can be downloaded from https://goo.gl/NVPQ7h. The meaning of a keyword "conductor" in the book with classification code precedes by $\mathrm{M}$ (Music) is most likely the person who leads a musical group. However, if the class is more specific to MT85 (Music - Instruction and study - Conducting. Score reading and playing) then the meaning of word "conductor" must be the person who leads a musical group.

From ontological point of view, class MT85 is related to Domain, Task, and Application Ontology. First level of class MT85 is M that refers to domain Music, the second level is T (MT) which associates to task Instruction and Study, and the third level is 85 (MT85) that refers to Application Ontology of conducting, score reading and playing. Although the class MT85 matches to ontology categorization, not all classes in LCC fulfil all ontology categorization. Some classes even have no Task Ontology.

\section{Expertise Representation Based on Bibliography}

\subsection{Multi-Domain Expertise Representation}

Based on their publications, most researchers have multi-domain (multi-discipline) expertise. For an example is Wisnu Jatmiko, a researcher from Faculty of Computer Science - University of Indonesia. He has published his works in some domains (subject areas): Computer Science, Engineering, Social Sciences, Mathematics, Genetics and Molecular Biology, Biochemistry, Medicine, Decision Sciences, Physics and Astronomy, Business, Management and Accounting, and Health Professions, as stated by Scopus.

To represent such expertise, a multi domain knowledge representation is needed. The representation should be as simple as possible that can be understood easily not only by human but also by machine. It has to be adopted from a proven knowledge representation and has been used widely. Ontology meets those requirements; it is simple and widely used as semantic web. Unfortunately, ontology by default is used to represent knowledge from a domain. It is not designed for multi-domain knowledge representation. Ontology can be used as expertise representation, but it has to be annotated first to make it multi-domain expertise representation. This process is well-known as ontology enrichment.

We proposed ontology enriched by LCC codes for multi-domain expertise representation. The proposed enriched ontology is developed by annotating the concepts and/or the relations by using LCC codes.

Fig. 1 shows enriched ontology of our proposal. The diagrams represent these sentences: (a) Harry is a conductor, (b) conductor holds stick made of metal, (c) Harry is singer with Metal genre, (d) metal is a conductor, and (e) metal conducts electricity. There are two words that have more than 1 definition depends on its domain. The first word is metal with LCC codes T, TN and M. This word in domain of Technology (T) and Metallurgy (TN) means a solid, shiny and hard material that conducts heat and electricity. In domain music, metal is a type of music. The other word is Conductor whose definition has been discussed in the previous section. 
Someone's expertise can be predicted by his/her publications. To determine the expertise based on documents published, we should analyse metadata of the publications. The metadata among others are: document title, author keywords, indexed keywords, main heading, and LCC code. Those metadata except LCC code can be retrieved from WorldCat web site (worldcat.org) freely, by using search engine provided. LCC code can be searched using the search engine in classify.oclc.org. We do not explain the prediction of someone's expertise here.

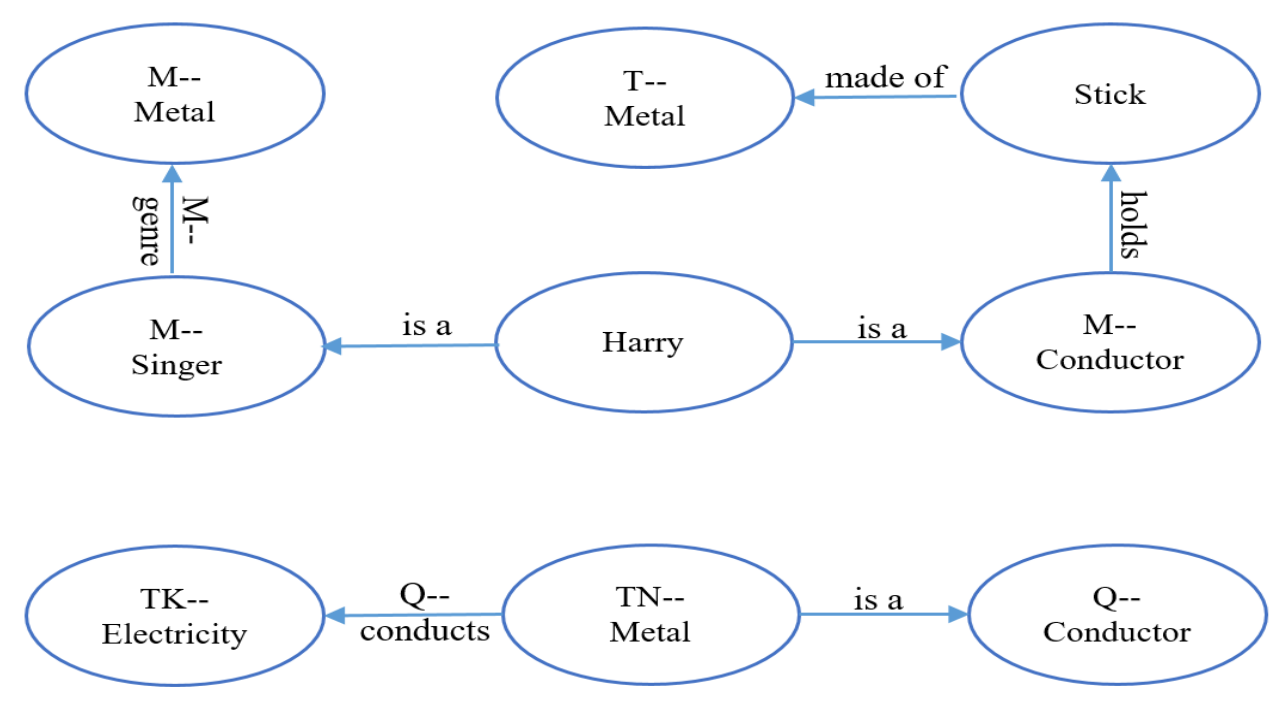

Fig. 1. Ontology enriched/annotated using LCC codes.

\subsection{Semantic Integration}

Semantic integration related to ontology is the process of interrelating concepts from different ontology. The process is very costly because it can only be done manually. Automatic semantic integration for multi domain ontology is not reliable. However, for single domain the precision and recall of automatic semantic integration can reach $92 \%$ and $96 \%$ respectively [65].

Semantic integration of multi-domain enriched ontology we proposed can be done automatically similar to single domain. The two ontologies in

Fig. 1 lied on two domains, technology domain and music domain, but can be integrated easily because the concepts has been annotated to distinguish each other. As can be seen in the figure, the two ontologies can be integrated because the two domains have interrelated concepts that is Metal (LCC code TN and T). If the ontologies have not been annotated, the two ontologies can make an invalid ontology if integrated semantically. This is caused by overlapping interpretation since metal in the music domain can be interrelated to metal in the metallurgy domain and conductor in the physics domain can be interrelated to conductor in the music domain.

Semantic integration is intended to generate new knowledge. The integration of the two ontologies in

Fig. 1 has interrelated TN--Metal and T--Metal to generate the following sentence: stick made of metal conducts electricity. Consequently, Harry should be careful while holding the stick because he may get electric shock. It seems that the new knowledge is obvious, because the sample ontologies are chosen as simple as possible.

\subsection{Limitations}

The proposed knowledge/expertise representation has some fundamental challenges to be implemented. The first is LCC codes of the concepts and the relationships of the ontology should be known. Currently, there is no reliable automatic document classification using LCC classes. Frank \& Paynter got maximum accuracy of 38\% from their experiments on automatic document classification [66] and Ávila-Argüelles et.al. got accuracy 36\% [67]. The largest accuracy was achieved by Larson [68], 46.6\%, but his experiments used only class "Z", 1 of 21 available classes in LCC.

Manually assigning LCC code to documents is very costly. To conduct document classification we have to know the main subject of the document to be classified. The classifier should be an experienced librarian who is used to classify books using LCC codes. Even for experienced librarian, it will take a while to look for an appropriate LCC code for a document. It will take much more time to classify concepts and relationships in an ontology, because a book only needs one class while an ontology needs many classes depending on the number of the sentences in the ontology.

The second challenge in implementing our proposed expertise representation is choosing the level of LCC code for concepts in the ontology. Fig. 1. shows concept "conductor" with different LCC code levels. One is in first level, LCC code T, and the other is in the second level, LCC code TN. LCC code T is for Technology and TN is for Metallurgy. 
The latter is more specific than the first one, but for ontology the more general classes is the better. For the case of conductor we should choose T instead of TN. Terms for concepts and relations are more precise if using a more general LCC code, and the upper ontology which consists of very general terms need no LCC code.

\subsection{Implementation}

The most suitable implementation of the proposed knowledge/expertise representation is to represent knowledge or expertise retrieved from documents in which the LCC codes of the documents are already known. Although the LCC code of the document has already been known, the retrieved ontology does not necessarily use the code to all concepts from the document. For illustration, we present a sentence "A mouse cable has been bitten by a mouse.". The mouse words have different meaning and it is possible to have different LCC codes. We suggest to use the LCC code of the document only for concepts provided by librarian and the author. Concepts from librarian usually are controlled vocabularies. Concepts provided by the author, despite not controlled vocabularies, are key concepts of the document. The concepts provided by librarian and also the author among others are listed in book indices, author keywords, indexed keywords, and subject headings.

\section{Conclusion and Future Works}

Someone by nature has expertise in multiple subject areas or multiple domains. Engineers are not only knowledgeable about manufacturing only. They normally also have other expertise in other subject areas like information technology, material science, business, and/or any other else. This research has answered the research question of how to represent knowledge/expertise in multi-domain environment.

The expertise suited to the proposed representation is expertise which is retrieved from document which the LCC code has already been known. It is expected to increase the accuracy of multi-domain semantic integration that can be done automatically with high precision and recall. Reasoning also can be implemented to the expertise representation because the representation is using ontology.

Following this research, there are some advanced researches needed. First is research on how to retrieve expertise from bibliography. There are several ways to do it such as retrieving expertise from millions of online documents in the forms of books, proceeding papers and journal papers. Secondly, the result from the first suggestion can be used for a research in expert finding by utilizing learning to rank methods. Thirdly, since the prerequisite of the proposed expertise representation is LCC code for the documents, it is necessary to do research on automatic documents classification using LCC codes.

\section{Acknowledgments}

Part of this research was funded by PITTA Grants (hibah Publikasi Internasional Terindeks untuk Tugas Akhir) fiscal year 2017 from Universitas Indonesia.

\section{References}

[1] Islam, M. \& Ikeda, M. (2014). Convergence issues of knowledge management in digital libraries: Steps towards state-of-the-art digital libraries. VINE, pp. 140-159.

[2] Berners-Lee, T. (1998). Semantic Web Road map. World Wide Web Consortium.

[3] Taye, M. M. (2010). Understanding Semantic Web and Ontologies: Theory and Applications. Journal of Computing, vol. 2, no. 6, pp. 182-192.

[4] Chen, R.-C., Huang, C.-Y. \& Ting, Y.-H. (2017). A chronic disease diet recommendation system based on domain ontology and decision tree. Journal of Advanced Computational Intelligence and Intelligent Informatics, vol. 21, no. 3, pp. 474-482.

[5] Corral, M., Antonelli, L. \& Sánchez, L. (2017). Health Ontology and Information Systems: A Systematic Review. IEEE Latin America Transactions, vol. 15, no. 1, pp. 103-120.

[6] Pozza, G., Borgo, S., Oltramari, A., Contalbrigo, L. \& Marangon, S. (2017). Information and organization in public health institutes: An ontology-based modeling of the entities in the reception-analysis-report phases. Journal of Biomedical Semantics, vol. 7, no. 1.

[7] Ait-Ameur, Y., Baron, M., Bellatreche, L., Jean, S. \& Sardet, E. (2017). Ontologies in engineering: the OntoDB/OntoQL platform. Soft Computing, vol. 21, no. 2, pp. 369-389.

[8] Zhou, J., Liu, J. \& Yang, H. (2016). Dynamic ontology for engineering knowledge management of complex product research \& development. Journal of Computer-Aided Design and Computer Graphics, vol. 28, no. 11, pp. 19571964.

[9] Gonzalez-Perez, C., Henderson-Sellers, B., McBride, T., Low, G. \& Larrucea, X. (2016). An Ontology for ISO software engineering standards: 2) Proof of concept and application. Computer Standards and Interfaces, vol. 48, pp. 112-123. 
[10] Dermeval, D., Vilela, J., Bittencourt, I., Castro, J., Isotani, S., Brito, P. \& Silva, A. (2016). Applications of ontologies in requirements engineering: a systematic review of the literature. Requirements Engineering, vol. 21, no. 4, pp. 405-437.

[11] García, M., García-Nieto, J. \& Aldana-Montes, J. (2016). An ontology-based data integration approach for web analytics in e-commerce. Expert Systems with Applications, vol. 63, pp. 20-34.

[12] Nederstigt, L., Aanen, S., Vandic, D. \& Frasincar, F. (2014). FLOPPIES: A Framework for Large-Scale Ontology Population of Product Information from Tabular Data in E-commerce Stores. Decision Support Systems, vol. 59, no. 1, pp. 296-311.

[13] Rosaci, D. \& Sarnè, G. (2014). Multi-agent technology and ontologies to support personalization in B2C ECommerce. Electronic Commerce Research and Applications, vol. 13, no. 1, pp. 13-23.

[14] Gonźalez-Conejero, J., Figueroa, R., Mũnoz-Gomez, J. \& Teodoro, E. (2014). Organized crime structure modelling for european law enforcement agencies interoperability through ontologies. Lecture Notes in Computer Science, pp. 217-231.

[15] Hage, J. \& Verheij, B. (1999). The law as a dynamic interconnected system of states of affairs: A legal top ontology. International Journal of Human Computer Studies, vol. 56, no. 1, pp. 1043-1077.

[16] Merriam-Webster. (2017). Merriam-Webster Dictionary, Available from: https://www.merriam-webster.com/ dictionary/reasoning Accessed 2017-07-24.

[17] Maree, M. \& Belkhatir, M. (2015). Addressing semantic heterogeneity through multiple knowledge base assisted merging of domain-specific ontologies. Knowledge-Based Systems, vol. 73, pp. 199-211.

[18] Pai, F.-P., Yang, L.-J. \& Chung, Y.-C. (2017). Multi-layer ontology based information fusion for situation awareness. Applied Intelligence, vol. 46, no. 2, pp. 285-307.

[19] Li, L., Wei, Y. \& Tian, F. (2017). A Framework for Ontology-Based Top-K Global Schema Generation. Journal on Data Semantics, vol. 6, no. 1, pp. 31-53.

[20] Dictionary.com. Expert, Available from: http://www.dictionary.com/browse/expert Accessed 2017-07-24.

[21] Dictionary.com. Expertise, Available from: http://www.dictionary.com/browse/expertise?s=t Accessed 2017-07-24.

[22] Horn, K. Van. (2017). From propositional logic to plausible reasoning: A uniqueness theorem. International Journal of Approximate Reasoning, vol. 88, pp. 309-332.

[23] Chajda, I. \& Kolařík, M. (2017). Reduced axioms for the propositional logics induced by basic algebras. Soft Computing, pp. 1-5.

[24] Caridroit, T. K. S. M. P. (2017). Contraction in propositional logic. International Journal of Approximate Reasoning, vol. 80, pp. 428-442.

[25] YiZhoua \& YanZhang. (2017). A progression semantics for first-order logic programs. Artificial Intelligence, vol. 250, p. 58-79.

[26] Perikos, I., Grivokostopoulou, F., Kovas, K. \& Hatzilygeroudis, I. (2016). Automatic estimation of exercises' difficulty levels in a tutoring system for teaching the conversion of natural language into first-order logic. Expert Systems, vol. 33, no. 6, pp. 569-580.

[27] Paes, A., Zaverucha, G. \& Costa, V. (2017). On the use of stochastic local search techniques to revise first-order logic theories from examples. Machine Learning, vol. 106, no. 2, pp. 197-241.

[28] Bruynooghe, M., Denecker, M. \& Truszczyński, M. (2016). First-order logic with inductive definitions for modelbased problem solving. AI Magazine, vol. 37, no. 3, pp. 69-80.

[29] Figueira, S., Gorín, D. \& Grimson, R. (2014). Independence friendly logic with classical negation via flattening is a second-order logic with weak dependencies. Journal of Computer and System Sciences, vol. 80, no. 6, pp. 11021118 .

[30] Kelk, S., Ierse, L. van, Scornavacca, C. \& Weller, M. (2016). Phylogenetic incongruence through the lens of monadic second order logic. Journal of Graph Algorithms and Applications, vol. 20, no. 2, pp. 189-215.

[31] Elberfeld, M. G. M. T. T. (2016). Where first-order and monadic second-order logic coincide. ACM Transactions on Computational Logic, vol. 17, no. 4, p. 25.

[32] Masterman, M. (1961). Semantic Message Detection for Machine Translation, using an Interlingua, International Conference on Machine Translation of Languages and Applied Language Analysis, Teddington, UK.

[33] Knaus, T., Burns, C., Kamps, J. \& Foundas, A. (2017). Atypical activation of action-semantic network in adolescents with autism spectrum disorder. Brain and Cognition, vol. 117, pp. 57-64.

[34] Ching, T. \& Williams, M. (2017). Association splitting of the sexual orientation-OCD-relevant semantic network. Cognitive Behaviour Therapy, pp. 1-17.

[35] Griffis, J. C., Nenert, R., Allendorfer, J. B., Vannest, J., Holland, S., Dietz, A. \& Szaflarski, J. P. (2017). The canonical semantic network supports residual language function in chronic post-stroke aphasia. Human Brain Mapping, vol. 38, no. 3, pp. 1636-1658.

[36] Marván, M., Chrisler, J., Gorman, J. \& Barney, A. (2017). The meaning of menarche: A cross-cultural semantic network analysis. Health Care for Women International, pp. 1-12.

[37] Deyne, S. De, Elvevåg, B., Hui, C., Poon, V. \& Chen, E. (2016). Rich semantic networks applied to schizophrenia: A new framework. Schizophrenia Research, pp. 454-455. 
[38] Gutiérrez-Villar, B., Alcaide-Pulido, P. \& Carbonero-Ruz, M. (2017). How do pre-university students see private universities? An exploratory study by combination of natural semantic networks and multidimensional unfolding. Revista de Investigacion Educativa, vol. 35, no. 2, pp. 519-535.

[39] Mosharraf, M., Taghiyareh, F. \& Alaee, S. (2017). Investigating elearning research trends in Iran via automatic semantic network generation. Journal of Global Information Technology Management, vol. 20, no. 2, pp. 91-109.

[40] Kwon, K., Bang, C., Egnoto, M. \& Rao, H. Raghav. (2016). Social media rumors as improvised public opinion: semantic network analyses of twitter discourses during Korean saber rattling 2013. Asian Journal of Communication, vol. 26, no. 3, pp. 201-222.

[41] Ertek, G., Tokdemir, G., Sevinç, M. \& Tunç, M. (2017). New knowledge in strategic management through visually mining semantic networks. Information Systems Frontiers, vol. 19, no. 1, pp. 165-185.

[42] Kim, J., Yoo, M., Lee, K. \& Seo, H. (2017). The innovation of the internet: a semantic network analysis of the Internet of Things. Asian Journal of Technology Innovation, vol. 25, no. 1, pp. 129-139.

[43] Agrawal, R., Imielinski, T. \& Swami, A. (1993). Mining Association Rules between Sets of Items in Large Databases, Proceedings of the 1993 ACM SIGMOD Conference, Washington.

[44] Agrawal, R. \& Srikant, R. (1994). Fast Algorithms for Mining Association Rules, International Conference on Very Large Data Bases, San Francico.

[45] Sowa, J. F. (1976). Conceptual Graph for for a Data Base Interface. IBM Journal of Research and Development, vol. 20, no. 4, pp. 336-357.

[46] Achsan, H. T. Y. \& Barcah, Q. K. (2015). Indonesia Coal Trade System: A knowledge-based application software. Energy Procedia, vol. 100, no. C, pp. 622-629.

[47] Ruiz, J., Arjona, M., Maña, A. \& Rudolph, C. (2017). Security knowledge representation artifacts for creating secure IT systems. Computers and Security, vol. 64, pp. 69-91.

[48] Kobrinskii, B. (2016). Approaches to the construction of cognitive linguistic-image models of knowledge representation for medical intelligent systems. Scientific and Technical Information Processing, vol. 43, pp. 289295.

[49] Achsan, H. T. Y. (2011). Web mining based on user profile and preferences, International DAAAM Symposium, Vienna.

[50] Fang, W., Guo, Y., Liao, W. \& Wang, F. (2016). Knowledge representation and annotation method based on ontology for complex products' design. Jisuanji Jicheng Zhizao Xitong/Computer Integrated Manufacturing Systems, CIMS, pp. 2063-2071.

[51] Gruber, T. R. (1993). Toward Principles for the Design of Ontologies Used for Knowledge Sharing. International Journal Human-Computer Studies, vol. 43, pp. 907-928.

[52] Ghose, A. (1976). Semantics of lisp and the ontology of functions. Kybernetes, vol. 5, no. 2, pp. 97-99.

[53] Ghose, A. (1978). Remarks on theories of computation and semantics of programming languages. Kybernetes, vol. 7, no. 4, pp. 301-304.

[54] Guarino, N. (1995). Formal ontology, conceptual analysis and knowledge representation. International Journal of Human-Computer Studies, vol. 43, pp. 625-640.

[55] O'Leary, D. E. (1998). Using AI in knowledge management: Knowledge bases and ontologies. IEEE Intelligent Systems and Their Applications, vol. 13, no. 3, pp. 34-39.

[56] Gruber, T. R. (1993). A Translation Approach to Portable Ontology Specification. Knowledge Acquisition, vol. 5, pp. 199-220.

[57] Musen, M. A. (1992). Dimensions of knowledge sharing and reuse. Computers and Biomedical Research, vol. 25, pp. $435-467$.

[58] Zaino, J. Schema.Org: The Fire's Been Lit. 15 September 2014, Available from: http://www.dataversity.net/schemaorg-fires-lit/ Accessed 2017-August-27.

[59] Cardoso, J. (2007). The Semantic Web Vision: Where are We?. IEEE Intelligent Systems, pp. 22-26.

[60] Wikipedia. Scopus, Available from: https://en.wikipedia.org/wiki/Scopus Accessed 2017-08-28.

[61] OCLC. WorldCat. OCLC Accessed 2017-08-28.

[62] Achsan, H. T. Y. \& Wibowo, W. C. (2014). A fast distributed focused-web crawling. Procedia Engineering, vol. 69, pp. 492-499.

[63] Faraj, S. \& Sproull, L. (2000). Coordinating Expertise in Software Development Teams. Management Science, pp. $1554-1568$.

[64] R.Puerta, H. E. A. \& A.Musen, M. (1994). Generation of knowledge-acquisition tools from domain ontologies. International Journal of Human-Computer Studies, vol. 41, no. 3, pp. 425-453.

[65] Shi, L., Li, S., Yang, X., Qi, J., Pan, G. \& Zhou, B. (2017). Semantic Health Knowledge Graph: Semantic Integration of Heterogeneous Medical Knowledge and Services. BioMed Research International, vol. 2017.

[66] Frank E. \& Paynter, G. W. (2004). Predicting Library of Congress Classifications From Library of Congress Subject Headings. Journal of The American Society for Information Science and Technology.

[67] Ávila-Argüelles, R., Calvo, H., Gelbukh, A. \& Godoy-Calderön, S. (2010). Assigning Library of Congress Classification codes to books based only on their titles. Informatica (Ljubljana), vol. 34, no. 1, pp. 77-84.

[68] Larson, R. R. (1992). Experiments in Automatic Library of Congress Classification. Journal of American Society for Information Science, pp. 130-148. 